\title{
Curcumin loaded in bovine serum albumin-chitosan derived nanoparticles for targeted drug delivery
}

\author{
SRIDHAR SKYLAB RAJAN ${ }^{1, *}$, AKILA PANDIAN ${ }^{1}$ and TAMILSELVI PALANIAPPAN ${ }^{2}$ \\ ${ }^{1}$ Biomedical Engineering Division, Department of ECE, CEG Campus, Anna University, Chennai 600025, India \\ ${ }^{2}$ Department of Anatomy, Sathyabama Dental College, Chennai 600119, India
}

MS received 14 September 2015; accepted 5 January 2016

\begin{abstract}
The main aim of this study is to prepare biocompatible polymeric nanoparticles for targeted delivery of curcumin to human colorectal adenocarcinoma (DLD-1) cells. Curcumin has an ability to block proliferation of cancer cells by suppressing the nuclear transcription factor NF-KB hence, it is chosen as drug in the current study. To avoid its low bio-availability, high dosage and poor aqueous solubility, curcumin nanoparticles are prepared and loaded in naturally available biopolymers like chitosan and bovine serum albumin (BSA) by nanoprecipitation method at pH 6.3. The prepared nanoformulation was then characterized for surface morphology, particle size, polydispersity index, FT-IR spectra, UV-Visible spectrometer, confocal microscopy and in vitro cytotoxicity studies. Results showed that sizes of the prepared nanoparticles were ranged between 181 and $363 \mathrm{~nm}$ and curcumin-loaded particles were selectively targeting colorectal carcinoma cells effectively when concentration gets increased. So this study proved that BSA-chitosan based nanoparticles can be used as an efficient vehicle for effective curcumin delivery in treatment of cancer cells.
\end{abstract}

Keywords. Nanoparticles; passive targeting; chitosan; curcumin; cancer.

\section{Introduction}

A typical drug delivery system involves delivering therapeutic agents to the targeted sites. To achieve the desired therapeutic response and to reduce the toxic side effects, the drug must be encapsulated within the nanoparticles [1]. An effective drug delivery system must be incorporated in polymeric nanocarriers for increasing the circulation time of the therapeutic drugs and also to control the high dosage levels [2,3]. Natural polymeric carriers are the widely chosen candidates for the targeted drug delivery systems due to their versatile aspects of nature like biocompatibility, biodegradability, reduced toxicity and low immunological properties.

Biodegradable polymeric nanoparticles hold great promise in targeted drug delivery due to their drug targeting specificity, improved bioavailability and sustained release of drugs with minimal toxicity and immunogenicity [4]. Chitosan, a natural polysaccharide, obtained from chitin is the widely used polymer of choice due to its hydrophilic characteristics. These polysaccharides are highly stable, non-toxic and biodegradable. Antitumoral, antibacterial and mucoadhesive aspects of chitosan make it an ideal candidate for drug delivery [5-8]. Bovine serum albumin (BSA), a natural biocompatible protein having non-toxic and non-antigenic characteristics can be used as a carrier of Paclitaxel for liver cancer treatment. Also, the BSA nanoparticles find applications in treating cerebral ischaemia by delivering Tanshinone

\footnotetext{
*Author for correspondence (sridharskylab@gmail.com)
}

IIA (TIIA). The nanoparticles loaded with 5-FU are prepared from chitosan and BSA which can be effectively used for liver cancer treatment [9-11].

Curcumin, an extract obtained from the rhizome of the plant Curcuma longa (Zingiberaceae) can be used as a model drug. The biological attributes of curcumin include anti-oxidant, anti-inflammatory and anti-cancer properties. Curcumin blocks the cell proliferation, induce apoptosis in tumour cells and block the nuclear kappa B (NF-KB) in various human cancer cell lines. Also faster degradation of curcumin at basic $\mathrm{pH}$ finds its applications for treating cancer at GI tract [12,13]. Nevertheless, the limiting factor of curcumin is its poor water solubility, which in turn reduces its bioavailability. And hence, to improve the level of low availability, polymeric nanocarriers were chosen.

The present work reveals the synthesis of curcuminloaded BSA-chitosan nanoparticles by nanoprecipitation method. The prepared nanoparticles were characterized for particle size distribution based on intensity, polydispersity index, scanning electron microscopy, FTIR spectroscopy UV-Visible spectrophotometer and confocal microscopy. The biocompatibility of the drug-loaded nanoparticles was evaluated using in vitro cytotoxicity studies.

\section{Materials and methods}

Chitosan, bovine serum albumin, Tween-20 and Tween-80 were purchased from SRL Chemicals, India. Curcumin was purchased from Sigma-Aldrich, India. 


\subsection{Nanoparticles preparation}

BSA-chitosan nanoparticles were prepared by nanoprecipitation method. Initially, $2.5 \%$ (w/v) of chitosan is dissolved in $1 \%$ acetic acid and $2 \%(\mathrm{w} / \mathrm{v}) \mathrm{BSA}$ dissolved in distilled water. The solvent phase containing chitosan-BSA solutions, tween-20 and acetone were added with drug curcumin (100 mg) under continuous stirring. Solvent phase was added into non-solvent phase containing tween-80 and $50 \mathrm{ml}$ distilled water under magnetic stirring. As solvent gets diffused into the aqueous phase, the chitosan gets precipitated and results in the instantaneous formation of nanoparticles. The BSA forms the core with encapsulated curcumin and $10 \mathrm{ml}$ of the prepared nanoparticle solutions were centrifuged at $16,000 \mathrm{rpm}$ for $30 \mathrm{~min}$ at $4^{\circ} \mathrm{C}$. The supernatant was discarded and pellet was washed three times with distilled water and lyophilized for $24 \mathrm{~h}$. The nanoparticles were then prepared from polymer loaded with and without drug in different combinations (see table 1).

\subsection{Surface morphology and particle size}

Scanning electron microscopy was performed to characterize the surface morphology of the curcumin loaded BSAchitosan nanoparticles. The particle size distribution based on intensity and polydispersity index of the drug-loaded nanoparticles were determined by dynamic laser scattering (DLS) using a Malvern system (Malvern Instruments).

\subsection{Fourier transform-infrared (FTIR) spectroscopy}

FTIR spectroscopy was done to study the interaction between the encapsulated drug and the nanoparticles. The samples (chitosan, BSA, curcumin, curcumin-loaded BSA-chitosan nanoparticles) were analysed using FTIR spectrometer. Spectral scanning was performed in the wavelength region between 4000 and $400 \mathrm{~cm}^{-1}$ at a resolution of $4 \mathrm{~cm}^{-1}$ with a scan speed of $2 \mathrm{~mm} \mathrm{~s}^{-1}$.

\subsection{Drug distribution within the nanospheres}

The distribution of curcumin within the nanospheres was examined using confocal microscope. Free nanoparticles, nanocurcumin, and PEGylated nanocurcumin were used in

Table 1. Concentrations of chitosan, BSA and curcumin (w/v).

\begin{tabular}{lccc}
\hline Model & $\begin{array}{c}\text { PEGylated } \\
\text { chitosan }(\mathrm{mg})\end{array}$ & $\begin{array}{l}\text { BSA } \\
(\mathrm{mg})\end{array}$ & Curcumin (mg) \\
\hline 1 & 250 & - & - \\
2 & - & 200 & - \\
3 & 250 & 200 & - \\
4 & 250 & 200 & 100 \\
\hline
\end{tabular}

this study. Since curcumin is naturally fluorescent in the visible green spectrum, no further labelling was used for confocal imaging. The samples were mounted on cover slips and visualized in a fluorescein isothiocyanate (FITC) channel and propidium iodide (PI) channel with excitation wavelength at 488 and $555 \mathrm{~nm}$.

\subsection{UV-Vis spectroscopy}

UV-Vis spectra of curcumin were recorded using a double beam Perkin Elmer-Lamda 35 instrument. A drop of the sample was taken and then added to acetone and placed in quartz cuvette which was considered as sample cuvette. In the other quartz cuvette, only the acetone was taken which was considered as reference cuvette.

\subsection{In vitro cytotoxicity studies: MTT assay}

The biocompatibility of the curcumin-loaded nanoparticles was evaluated using MTT assay. The cultured cells (H9C2 cardiomyocytes and DLD1 colorectal adenocarcinoma cells) were seeded in 96-well plates with cell density of 10,000 cells per well. Then, the cells were treated with different formulations (curcumin, BSA-chitosan nanoparticles and nanocurcumin) for $24 \mathrm{~h}$. Later, the cells were incubated with $10 \mu \mathrm{l}$ of $\operatorname{MTT}\left(5 \mathrm{mg} \mathrm{ml}^{-1}\right)$ for $4 \mathrm{~h}$ at $37^{\circ} \mathrm{C}$. The media was removed and $100 \mu \mathrm{l}$ of DMSO was added to each well. Viable cells cause the reduction of yellow tetrazolium salt to violet formazan crystal. Finally, the absorbance was measured at $590 \mathrm{~nm}$ using ELISA reader. Based on the cell viability percentage, the cytotoxic nature of the drug-loaded nanoparticles was evaluated $[15,16]$.

\section{Results and discussion}

\subsection{Surface morphology and particle size}

The drug-loaded nanoparticles exhibited a spherical morphology with mean diameter between 200 and $300 \mathrm{~nm}$ (figure 1). The result of the study was in agreement with the result obtained by Fan Yuan et al [8]. It ensured that prepared nanoparticles can efficiently reach the leaky tumour vasculature passively (EPR effect). The average size of the nanocurcumin was in agreement with the DLS results (nanoparticles with aggregates).

Polydispersity index values increases with the increase in concentration of polymers and drug. PDI values confirmed that the homogeneity of the drug-loaded nanoparticles decreases when compared with free nanoparticles (table 2).

\subsection{FTIR spectroscopy analysis}

FTIR spectra of chitosan, BSA, curcumin and curcuminloaded BSA-chitosan nanospheres were recorded by a FTIR 


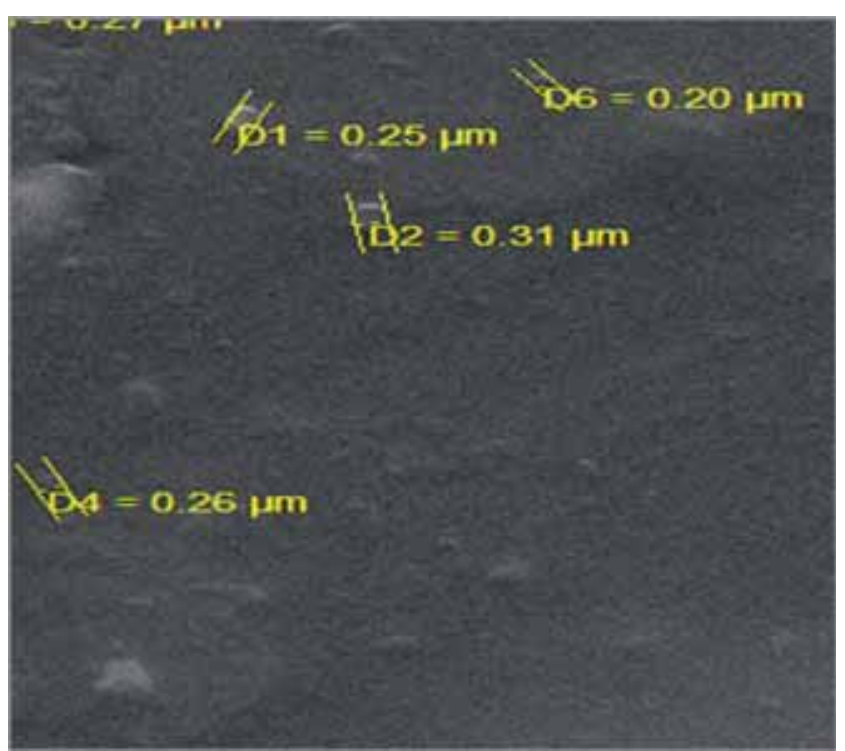

Figure 1. SEM image of curcumin-loaded nanoparticles (magnification $200 \mathrm{~nm}$ ).

Table 2. Average particle size and PDI.

\begin{tabular}{lcc}
\hline Model & Average particle size $(\mathrm{nm})$ & PDI \\
\hline 1 & 181.9 & 0.28 \\
2 & 149.4 & 0.44 \\
3 & 241.8 & 0.45 \\
4 & 363.6 & 0.61 \\
\hline
\end{tabular}

spectrometer (figures 2-6). The FTIR spectra of chitosan showed strong peaks at 3456.0, 1632.3, 1485.2 and $1415.3 \mathrm{~cm}^{-1}$ which correspond to $\mathrm{O}-\mathrm{H}$ stretching and hydrogen bonding, $\mathrm{N}-\mathrm{H}$ bonding of primary amines, $\mathrm{C}-\mathrm{C}$ aromatic stretch, respectively. The FTIR spectra of BSA showed characteristic peaks at $3447.0,2918.2,2849.6,2103.3$, $1636.4,1459.5,1292.8$ and $716.6 \mathrm{~cm}^{-1}$ due to $\mathrm{O}-\mathrm{H}$ stretching and hydrogen bonding, $\mathrm{C}-\mathrm{H}$ stretch, $-\mathrm{C}=\mathrm{C}-$ alkynes stretch, $\mathrm{N}-\mathrm{H}$ bonding of primary amines, $\mathrm{C}-\mathrm{C}$ aromatic stretch and $\mathrm{C}-\mathrm{H}$ bond, $\mathrm{N}-\mathrm{O}$ symmetric stretch, $\mathrm{C}-\mathrm{H}$ bonding vibrations, respectively. The FTIR spectra of curcumin showed characteristic peaks at 3621.6, 3570.2 and 3405.9, 2920.6 and $2850.1,1597.0,1292.2,1157.2 \mathrm{~cm}^{-1}$ that correspond to strong $\mathrm{O}-\mathrm{H}$ stretch, $\mathrm{O}-\mathrm{H}$ stretching and $\mathrm{H}$-bonded, $\mathrm{C}-\mathrm{H}$ stretch, $\mathrm{C}-\mathrm{C}$ stretch in aromatics (strong), $\mathrm{C}-\mathrm{O}$ stretching vibrations.

In the FTIR spectrum of drug-loaded nanoparticles (nanocurcumin), a shift from 3456.0 to $3399.0 \mathrm{~cm}^{-1}$ was observed and the valley of $3399.0 \mathrm{~cm}^{-1}$ became wider, thereby indicating enhanced hydrogen bonding. In nanocurcumin, the peak at $1636.4 \mathrm{~cm}^{-1}$ of $\mathrm{N}-\mathrm{H}$ bonding vibration shifted to $1597.0 \mathrm{~cm}^{-1}$. It was due to the interaction of aromatic group of curcumin with BSA-chitosan nanoparticles.

\subsection{Drug distribution within the nanospheres}

Free nanoparticles do not exhibit any fluorescence in FITC and PI channels. Curcumin-loaded nanoparticles (nanocurcumin and PEGylated nanocurcumin) exhibits green fluorescence (by curcumin auto-fluorescence) in FITC channel

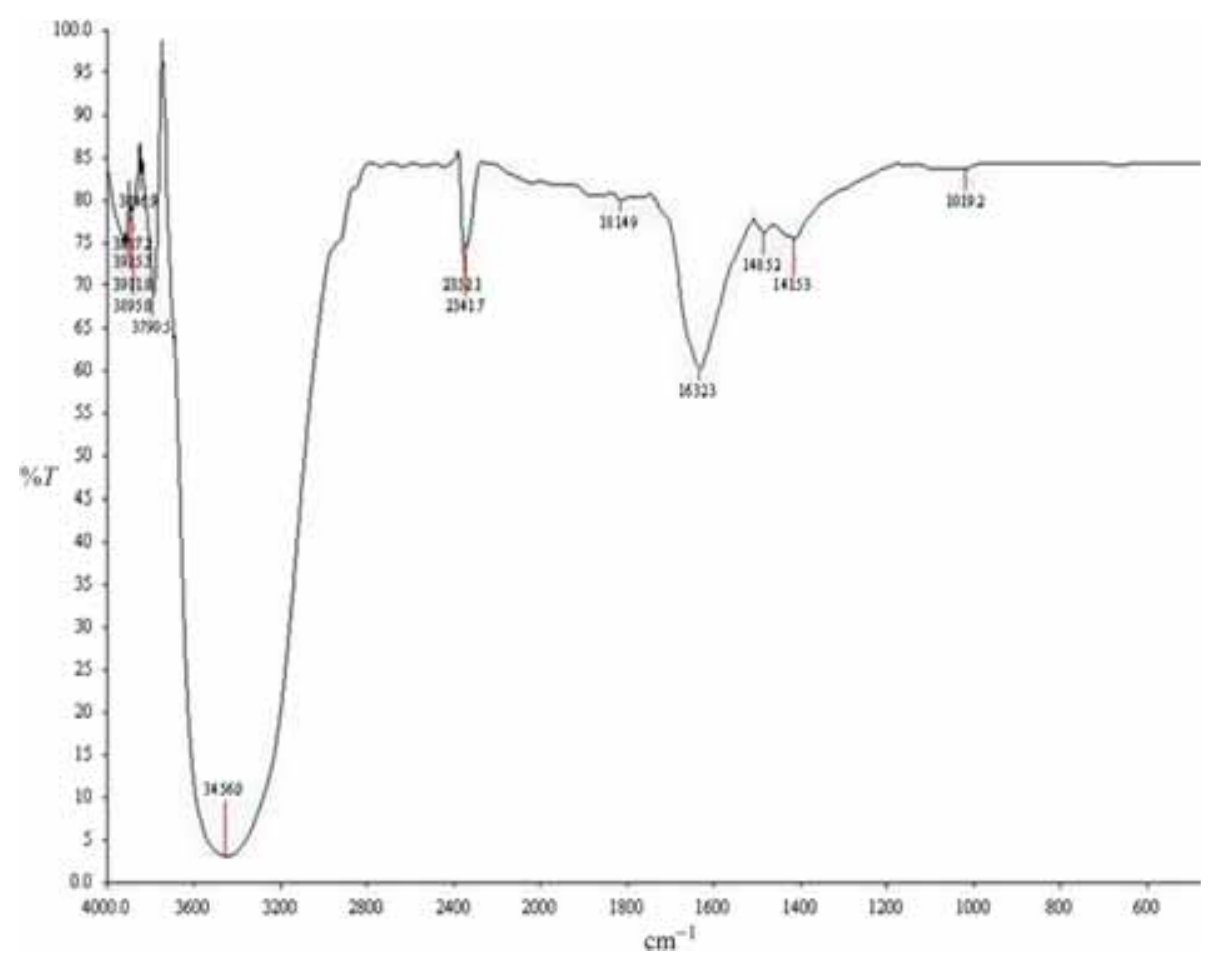

Figure 2. FTIR spectra of chitosan. 
(488 nm). But no fluorescent image obtained from PI channel for all the three samples (figure 7). This confirms the auto-fluorescence property of curcumin showing the green fluorescent image in FITC channel. The green fluorescent image confirms that curcumin is encapsulated within the nanoparticles.

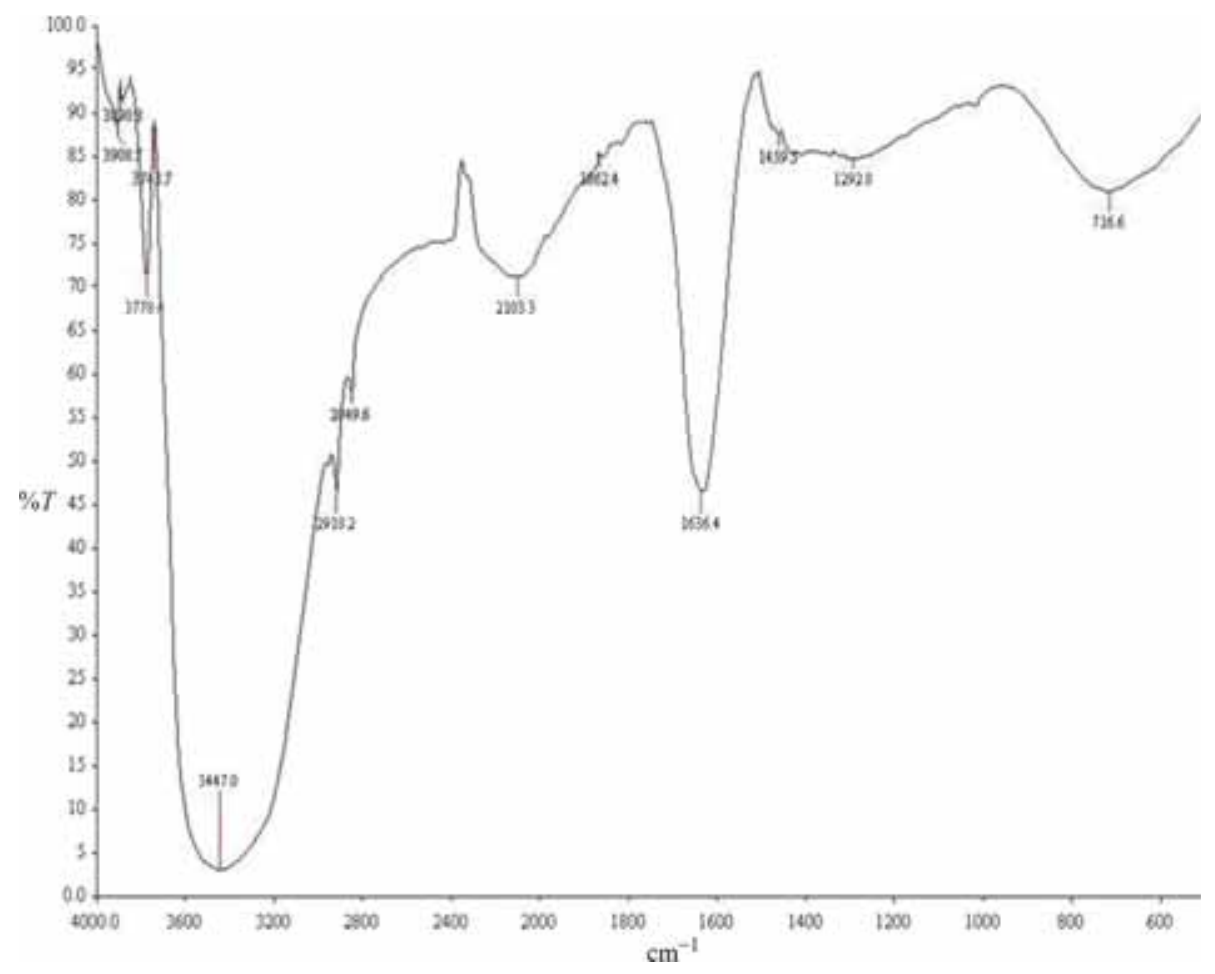

Figure 3. FTIR spectra of BSA.

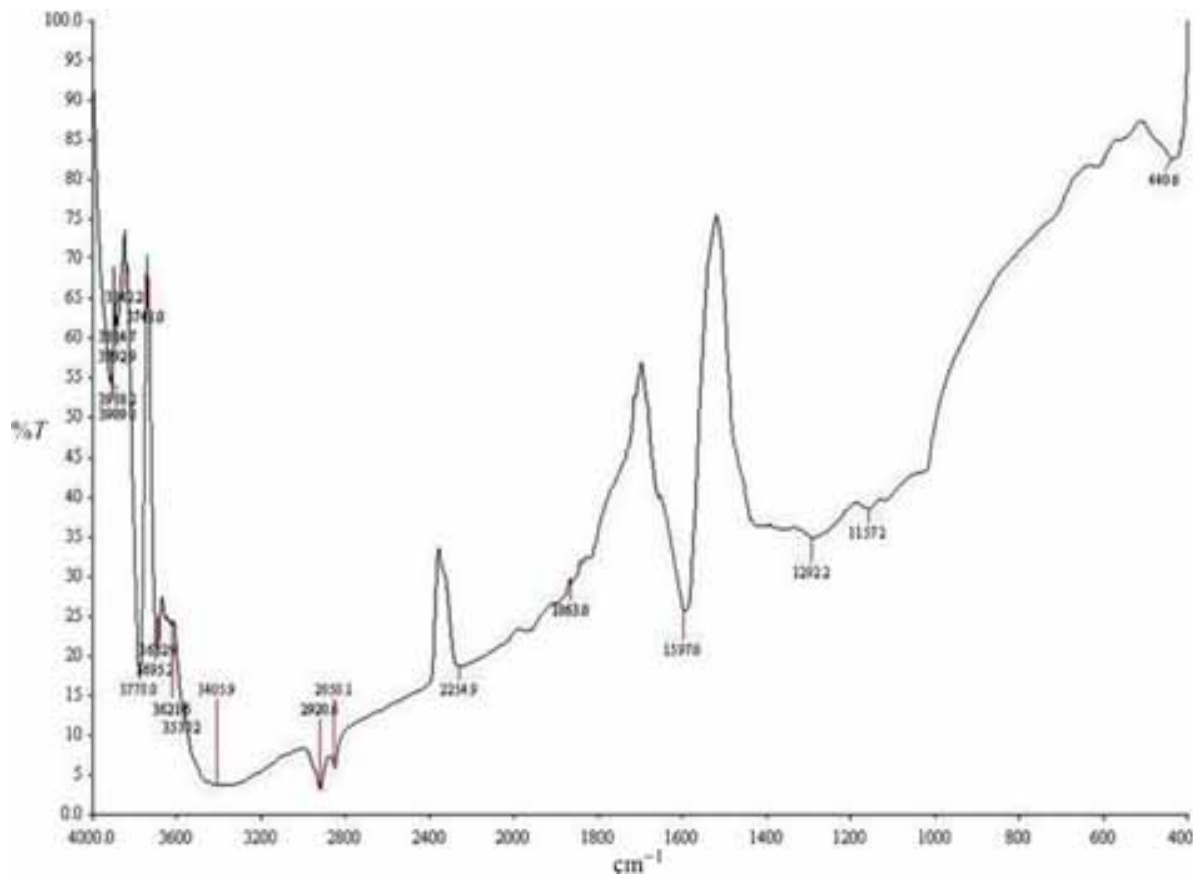

Figure 4. FTIR spectra of curcumin. 


\section{$3.4 \quad U V-V i s$ spectroscopy}

Curcumin (red) exhibits intense absorption in the visible region of UV-Vis spectra (422 nm). BSA-chitosan nanoparticles (blue) and PEGylated BSA-chitosan nanoparticles (green) showed similar absorption in the visible region with absorbance wavelength at 419.4 and $419.9 \mathrm{~nm}$. This confirms the existence of curcumin within the nanospheres.

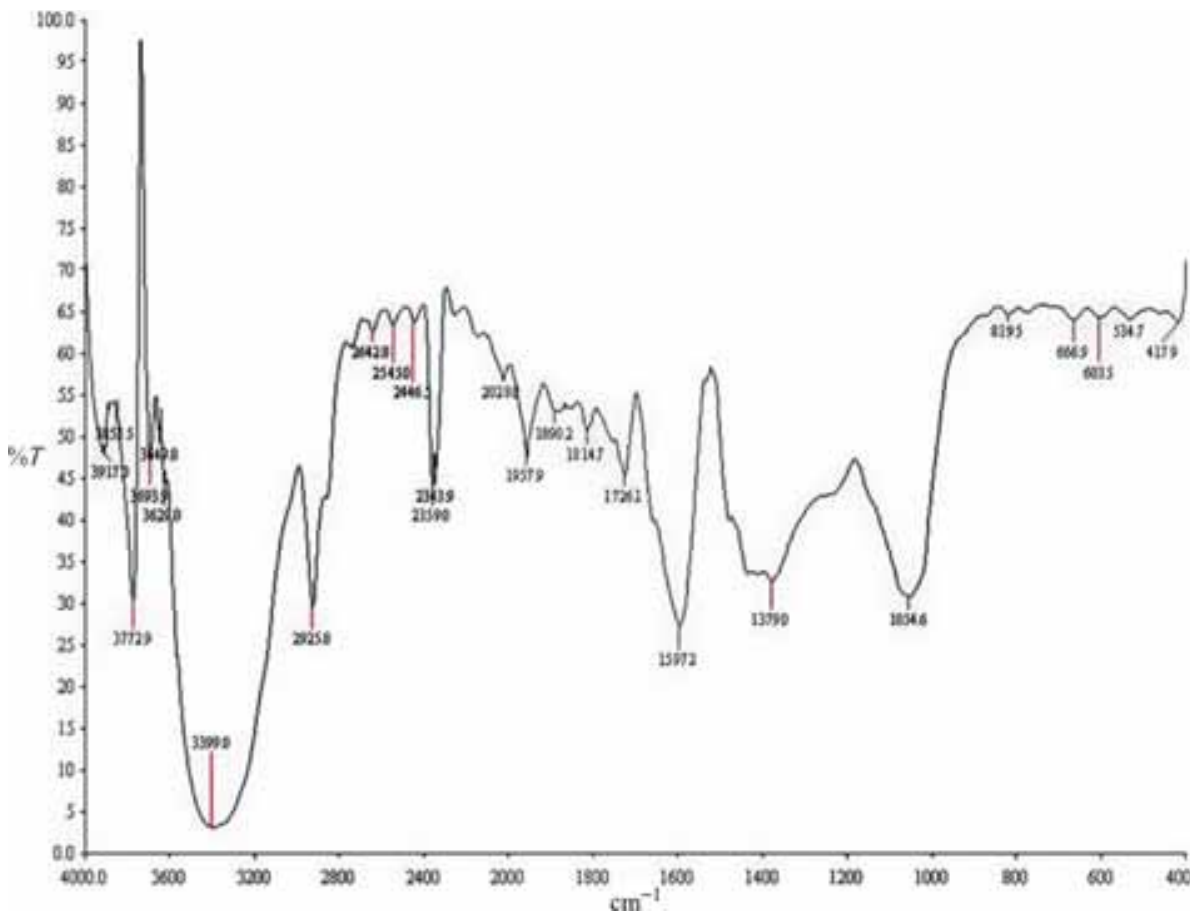

Figure 5. FTIR spectra of curcumin-loaded nanoparticles.

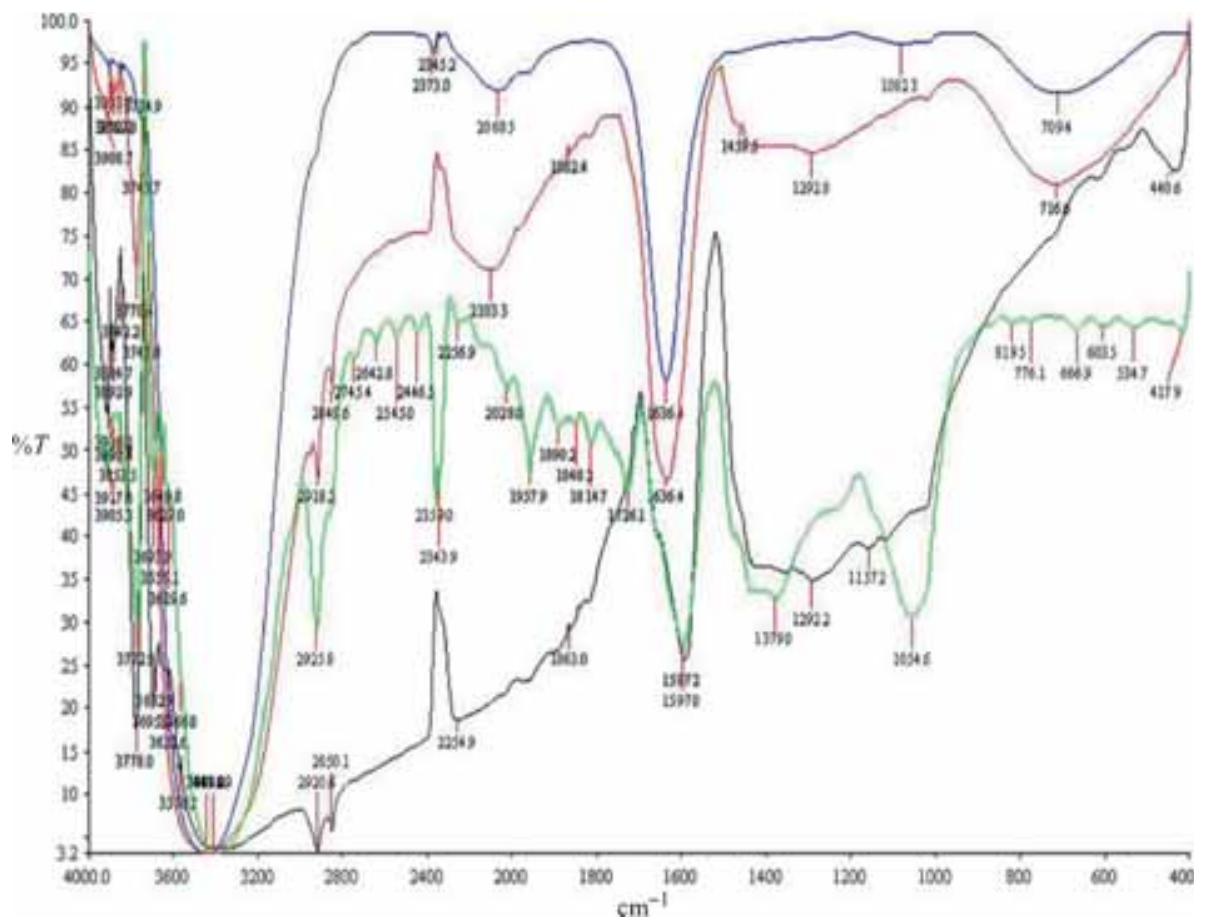

Figure 6. FTIR spectra overlay. 

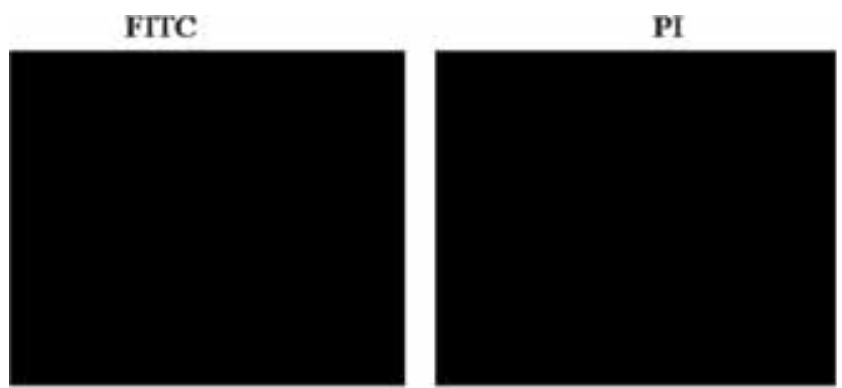

Bare nanoparticle
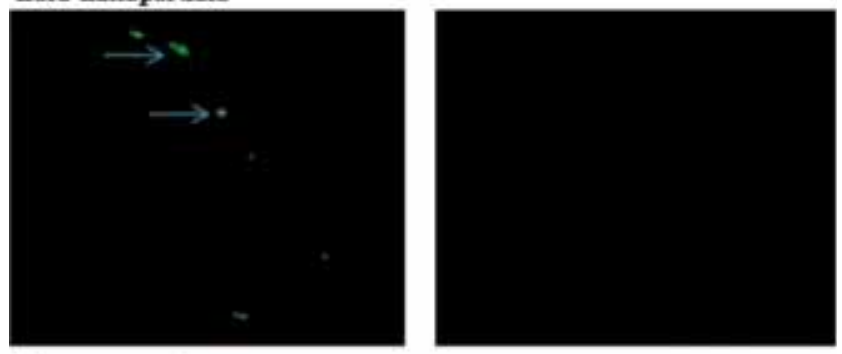

Nanocurcumin
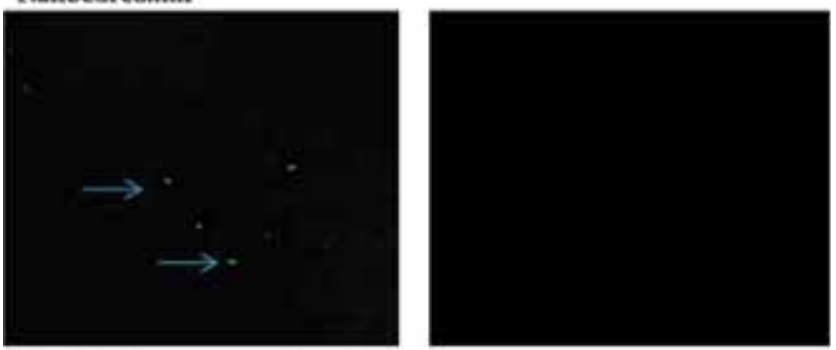

Pegylated nanocurcumin

Figure 7. Curcumin-loaded nanoparticles exhibiting green fluorescence (by curcumin auto-fluorescence) in FITC channel.

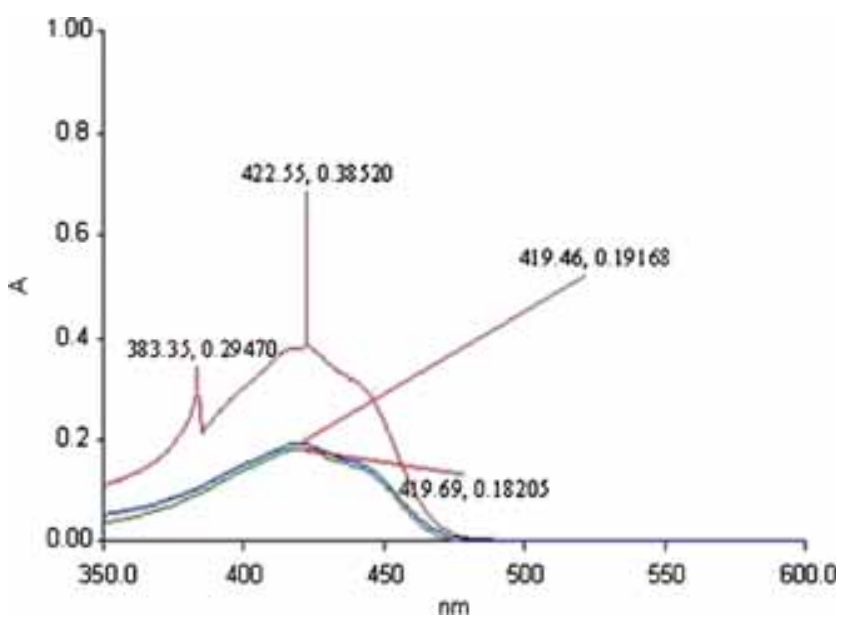

Figure 8. UV-Vis spectra of curcumin (red), nanocurcumin (blue) and PEGylated nanocurcumin (green) at pH 6.3.

Blank nanoparticles do not exhibit any absorbance in the visible region of UV-Vis spectra (figure 8).

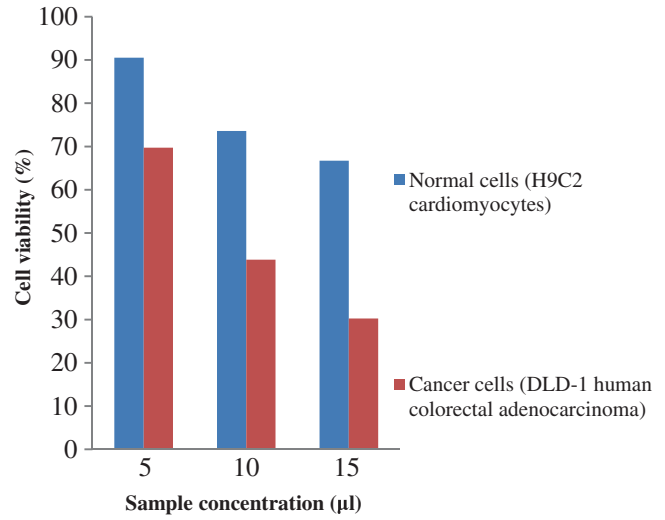

Figure 9. In vitro cytotoxicity studies using MTT assay after 24-h treatment.

\subsection{Cytotoxicity studies}

The biocompatibility of the prepared samples was evaluated by in vitro cytotoxic studies. MTT assay showed the viable nature of the living cells by the conversion of yellow tetrazolium salt into violet formazan crystal.

After $24 \mathrm{~h}$ treatment, the prepared PEGylated nanocurcumin showed cell viability of $91 \%$ on normal H9C2 cardiomyocytes and $70 \%$ of viability in colorectal adenocarcinoma cells for $5 \mu \mathrm{l}$ concentration. The viability percentage decreases with an increase in concentration of the nanoparticles. These results confirmed that the cytotoxicity was dosage-dependent and prepared nanoparticles were selectively toxic to DLD-1 cancer cells (figure 9).

\section{Conclusion}

This work proposes a new method of preparing curcuminloaded nanoparticles using chitosan (2.5\%) and BSA (2\%) by nanoprecipitation method. Nanoparticles prepared by this method have particle size in the range of nanometres (200-300 nm) can be used for passive method of drug targeting. Polydispersity index in the range of $0.2-0.6$. PDI values confirmed that the nanoparticles became poly-dispersive in nature with the increase in the concentration of polymers and drug.

Scanning electron microscopy results confirmed that the prepared nanoparticles had spherical morphology with an average diameter of the nanoparticles $(Z$ average $=258 \mathrm{~nm}$ ). FTIR spectral studies showed that the drug reacts with the polymers through enhanced intermolecular hydrogen bonding and interaction of amino and aromatic groups. Also, intermolecular hydrogen bonding reduces intramolecular hydrogen bonding of phenolic hydrogen. Confocal microscopy results proved that the auto-fluorescence (green fluorescence in FITC channel) property of curcumin which are loaded in BSA-chitosan nanoparticles. UV-Vis spectral studies confirmed the presence of curcumin in nanocurcumin $\left(\lambda_{\max }=419.4 \mathrm{~nm}\right)$ and PEGylated nanocurcumin 
$\left(\lambda_{\max }=419.6 \mathrm{~nm}\right)$. In vitro cytotoxicity studies revealed that the prepared nanocarrier was biocompatible (at low concentration of $5 \mu \mathrm{l}$ for $24 \mathrm{~h}$ treatment) showing greater viability for normal H9C2 cells (91\%) when compared to DLD-1 cancer cells $(70 \%)$. MTT assay showed that curcumin would not exhibit any cytotoxic effect on normal H9C2 cardiomyocytes even at high drug concentrations. Thus, BSA-chitosan based nanocarriers could be used as a pertinent vehicle to deliver curcumin.

\section{References}

[1] Abhishek Yadav, Vinay Lomash and Swaran J S Flora 2012 Chemico-Biol. Interact. 19949

[2] Anindita Mukerjee and Jamboor K Vishwanatha 2009 AntiCancer Res. 293867

[3] Anita R Dudhania and Shantha L Kosaraju 2010 Carbohyd. Polym. 81243

[4] Barnabas Wilson, Ambika T V, Dharmesh Kumar Patel R, Josephine Leno Jenita and Priyadarshini S R B 2012 Inter. J. Biol. Macromol. 51874

[5] Catarina Pinto Reis, Ronald J Neufeld, António J Ribeiro and Francisco Veiga 2006 Nanomed.: Nanotechnol. Biol. Med. 28
[6] Chun Li and Sidney Wallace 2008 Adv. Drug Deliv. Rev. 60 886

[7] Dasha M, Chiellini F, Ottenbrite R M and Chiellini E 2011 Progr. Polym. Sci. 36981

[8] Fan Yuan, Marc Dellian, Dai Fukumura, Leunig M, Berk D A, Torchilin V P and Jain R K 1995 Cancer Res. 553752

[9] Jae Hyung Park, Kwangmeyung Kim and Ick Chan Kwon 2010 Adv. Drug Deliv. Rev. 6228

[10] James W Lillard Jr and Rajesh Singh 2009 Exp. Molec. Pathol. 86215

[11] Jianing Qi, Ping Yao, Fen He, Yu Chuiliang and Chong Huang 2010 Inter. J. Pharmaceut. 393176

[12] Kumaravel M, Sankar P and Rukkumani R 2012 Eur. Rev. Med. Pharmacol. Sci. 161900

[13] Kumaresh S Soppimath, Tejraj M Aminabhavi, Anandrao R Kulkarni and Walter E Rudzinski 2001 J. Control. Rel. 701

[14] Mohammad Reza Ashrafi Kooshka, Kamran Mansouria, Mohammad Mostafa Nadib, Sina Khodarahmia and Reza Khodarahmi 2013 Appl. J. Rep. Pharmaceut. Sci. 266

[15] Mostafa Rahimnejad, Ghasem Najafpour and Gholamreza Bakeri 2012 Colloids Surf. A: Physicochem. Eng. Aspects 41296

[16] Sharma R A, Gescher A J and Steward W P 2005 Eur. J. Cancer 411955 